\title{
Role of Magnetic Resonance Imaging in diagnosis of Endometriosis
}

\author{
Sherif H. Abo-Gamra, Aliaa S. sheha, Alaa S. Abd-Elhafez, Marian A. Thabet \\ Radiology Department, Faculty of Medicine, Ain Shams University, Cairo, Egypt \\ Corresponding author: Marian A. Thabet, Mobile: +20124471682, E-mail: marianayad95@gmail.com
}

\begin{abstract}
Background: Endometriosis is defined as the presence of functional endometrial glands and stroma outside of the uterine cavity. Although laparoscopy is the standard of reference for diagnosis of endometriosis, reliable identification of the disease before laparoscopy would aid the gynecologist in choosing the preferred therapeutic approach, medical or surgical. Ultrasound is performed initially, but MRI is increasingly being used, particularly when sonographic findings are unclear, when deep pelvic endometriosis is suspected or when surgery is planned. Objective: This study aimed to evaluate the accuracy of Magnetic Resonance Imaging in diagnosis of Endometriosis. Patients and Methods: 30 Premenopausal female Patients with clinical and/or sonographic suspicion of endometriosis underwent pelvic MRI. All our imaging results were finally compared to the laparoscopic results with histopathologic verification which was our gold standard. The main outcome parameters, The Sensitivity, Specificity, positive predictive value (PPV), negative predictive value (NPV) and accuracy of MRI in diagnosing endometriosis were calculated. Results: The Sensitivity, Specificity, positive predictive value (PPV), negative predictive value (NPV) and accuracy of MRI in diagnosing endometriosis were $95.65 \%, 57.14 \%, 88 \%, 80 \%, 86.7 \%$ respectively. Conclusion: MRI is the best problem-solving tool as in cases of indeterminate adnexal findings on sonography, when deep infiltrating endometriosis is suspected, or for presurgical mapping.
\end{abstract}

Keywords: Endometriosis, MRI, Pelvis, Ultrasound, Laparoscopy

\section{INTRODUCTION}

Endometriosis is a common gynaecological condition affecting women of reproductive age, it is defined as the presence of functional endometrial glands and stroma outside of the uterine cavity. This ectopic tissue responds to hormonal changes resulting in cyclical haemorrhage and pain, giving the disease its clinical features ${ }^{(1)}$.

Its estimated prevalence varies between $2 \%$ and $10 \%$ within the general female population, although it may be as high as $50 \%$ in infertile women ${ }^{(2)}$.

Secondary dysmenorrhea, deep dyspareunia, sacral backache with menses, perimenstrual diarrhea, cramping and dyschezia, dysuria, and hematuria are the most common and relevant clinical manifestations ${ }^{(3)}$.

Laparoscopy is the standard of reference for diagnosis of endometriosis followed by histologic confirmation ${ }^{(4)}$.

Currently, ultrasound is preferred for the initial assessment of both endometriomas and deep pelvic endometriosis. However, transvaginal ultrasound, even with adequate bowel preparation and use of high-frequency probes has important limitations, because of the relatively small field-of-view and operator dependency ${ }^{(5)}$.
MRI is being increasingly used for the evaluation of endometriosis, with reported sensitivity and specificity values ranging from $69-92 \%$ and $75-98 \%$, respectively ${ }^{(5)}$.

The use of MRI offers the following advantages: Excellent at demonstrating the haemorrhagic content (new and old) of endometriomas. Used in identifying the presence of deeply-infiltrating endometriotic implants. Particularly useful in pre-operative assessment of disease distribution and the presence of adhesions, especially within the posterior compartment and pouch of Douglas which helps guide subsequent laparoscopic surgery. Vital as a problem-solving tool when assessing an ultrasound-indeterminate adnexal mass, helping differentiate ovarian cystic and solid lesions from endometriomas and also in the detection of malignant transformation within an endometrioma ${ }^{(6)}$.

\section{PATIENTS AND METHODS}

Patients: This is a retrospective study that included 30 female patients referred from the Gynecology Department to the Radiology Department (Women's imaging unit) of Ain Shams University Hospitals in the period from November 2017 to June 2018. The study was approved by the Ethics Board of Ain Shams University and 


\section{an informed written consent was taken from each participant in the study.}

\section{A- Inclusion criteria included:} Premenopausal female Patients with clinical and/or sonographic suspicion of endometriosis. Performance of a standardized laparoscopy with surgical ablation and histopathologic verification after MRI examination as a reference.

B- Exclusion criteria included: General contraindications against an MRI examination (i.e., claustrophobia and metallic implants). Postmenopausal status.

\section{All cases were subjected to the following:}

Full history taking with special emphasis on age, parity, age of menarche, past history of gynecological troubles or operations, menstrual history, and main complaint.

Routine laboratory investigation for all patients including $\mathrm{CBC}$, random blood sugar, liver functions and kidney functions.

Ultrasound examination: All patients underwent a preliminary pelvic ultrasound examination. The examination was performed using GE logic 7 ultrasound machine. Trans-abdominal and trans-vaginal approaches using $3-4 \mathrm{MHz}$ and 7-8 $\mathrm{MHz}$ probes respectively, were performed.

MR imaging using a 1.5-T MR imaging unit (Achieva, Philips medical system). All the patients were imaged in the supine position, head pointing to the magnet (HFS) using a high resolution body coil.

\section{MR Imaging analysis:}

Criteria for endometriosis in MRI were described by Bazot et al ${ }^{(7)}$, In short, low signal intensity lesions on T2W images, possibly associated with hyperintense lesions on T1W images and cystic regions were findings of endometriosis. T1W high signal intensity cysts possibly with wall thickening, fluid levels and reactions of the surrounding tissue were interpreted as positive for ovarian endometriosis. For peritoneal lesions high signal intensity spots on fat saturated T1W images were rated positive for endometriosis.

So MR images were analyzed for the following: Presence of ovarian cystic lesions. Whether one or both ovaries were involved. Signal intensity of the cyst on T1WIs, T2WIs and on the Fat suppression sequences. Presence of septations within the cyst. Presence of solid components. Enhancement of the solid component if present. Presence of adhesions. Screening the vesicouterine pouch, vesicovaginal septum, bladder, fallopian tubes, uterine ligaments, cul-de-sac, rectovaginal septum, anterior abdominal wall and bowel for endometriotic implants. All our imaging results were finally compared to the laparoscopic results with histopathologic verification which was our gold standard.

\section{RESULTS}

The patients age ranged from $22-48$ years with the mean age of $35.9 \pm 8.25$.

Clinical presentations were as follows: 17 were presented with pelvic pain $(56.7 \%), 6$ were presented with infertility (20\%), 4 were presented with dysmenorrhea (13.3\%), 2 were presented with Caesarean section scar palpable mass (6.7\%), and 1 was presented with Dyschezia (3.3\%) (Table 1).

Table (1): The number and percentage of different clinical presentation of the studied patients.

\begin{tabular}{|l|c|c|}
\hline \multicolumn{1}{|c|}{ Clinical presentation } & Number & Percentage \\
\hline Pelvic pain & 17 & $56.7 \%$ \\
\hline Infertility & 6 & $20 \%$ \\
\hline Dysmenorrhea & 4 & $13.3 \%$ \\
\hline $\begin{array}{l}\text { Caesarean section scar } \\
\text { palpable mass }\end{array}$ & 2 & $6.7 \%$ \\
\hline Dyschezia & 1 & $3.3 \%$ \\
\hline Total & 30 & $100 \%$ \\
\hline
\end{tabular}

Findings in cases diagnosed as endometriosis on MRI study: This group included $25 / 30$ patients diagnosed with endometriosis on MRI study $(83.3 \%)$. Their ages ranged from 22 to 48 years. In 22 of the 25 patients, endometriosis was found in the ovaries as ovarian cysts (88\%). In 2 of the 25 patients, endometriosis was located in the anterior abdominal wall (8\%). In 1 of the 25 patients, endometriosis was found on the rectal wall (4\%) (Table 2).

Table (2): Infiltration sites of cases diagnosed as endometriosis on MRI study.

\begin{tabular}{|l|c|c|}
\hline \multicolumn{1}{|c|}{ Infiltration site } & Number & Percentage \\
\hline Ovaries & 22 & $88 \%$ \\
\hline $\begin{array}{l}\text { Anterior abdominal } \\
\text { wall }\end{array}$ & 2 & $8 \%$ \\
\hline Rectal wall & 1 & $4 \%$ \\
\hline Total & 25 & $100 \%$ \\
\hline
\end{tabular}

On MRI, 6 out of 25 cases (24\%) showed relatively homogeneous high signal intensity (similar to or greater than that of fat) on T1WI \& on T2WI, 8 out of 25 cases (32\%) showed high 
signal intensity on T1WI whereas on T2WI they showed intermediate signal intensity, 4 out of 25 cases (16\%) showed high signal intensity on T1WI while on T2WI they showed low signal intensity, 4 out of 25 cases (16\%) showed low signal intensity on T1WI \& on T2WI, and 3 out of 25 cases (12\%) showed low signal intensity on T1WI whereas on T2WI they showed high signal intensity (Table 3).

All cases were not suppressed on fat suppression sequences indicating a hemorrhagic nature.

Table (3): Pattern of MRI signals of endometriotic lesions.

\begin{tabular}{|l|c|c|}
\hline \multicolumn{1}{|c|}{ MRI signal pattern } & Number & Percentage \\
\hline High T1, high T2 WIs & 6 & $24 \%$ \\
\hline $\begin{array}{l}\text { High T1, intermediate } \\
\text { T2 WIs }\end{array}$ & 8 & $32 \%$ \\
\hline High T1, low T2 WIs & 4 & $16 \%$ \\
\hline Low T1, low T2 WIs & 4 & $16 \%$ \\
\hline Low T1, high T2 WIs & 3 & $12 \%$ \\
\hline Total & 25 & $100 \%$ \\
\hline
\end{tabular}

Table (4): Results of MRI examination compared to histopathology.

\begin{tabular}{|c|c|c|}
\hline True positive 22 & False positive 3 & Diagnosed positive $=25$ \\
\hline False negative 1 & True negative 4 & Diagnosed negative $=5$ \\
\hline 23 & 7 & Total $=30$ \\
\hline
\end{tabular}

The Sensitivity, Specificity, positive predictive value (PPV), negative predictive value (NPV) and accuracy of MRI in diagnosing endometriosis were calculated and illustrated in the following table

Table (5): Sensitivity, Specificity, PPV, NPV and accuracy of MRI.

\begin{tabular}{|c|c|}
\hline Sensitivity & $95.65 \%$ \\
\hline Specificity & $57.14 \%$ \\
\hline PPV & $88 \%$ \\
\hline NPV & $80 \%$ \\
\hline Accuracy & $86.7 \%$ \\
\hline
\end{tabular}

\section{DISCUSSION}

Endometriosis is defined as the presence of functional endometrial glands and stroma outside of the uterine cavity primarily as implants in the ovaries and pelvic peritoneum $^{(1)}$.

The main clinical features of endometriosis are chronic pelvic pain and infertility ${ }^{(1)}$.

Laparoscopy is the gold standard for the diagnosis of endometriosis and the definitive diagnosis of endometriosis relies on histological confirmation of endometrial glands and stroma outside of the endometrial cavity $^{(4)}$.

Radiologists are often involved in the diagnosis and work-up of this disease in one of two scenarios: They are asked to exclude endometriosis in a woman with pelvic pain or infertility or they are considering endometriosis in the differential diagnosis of an adnexal mass ${ }^{(8)}$.

Ultrasound is preferred for the initial assessment of both endometriomas and deep pelvic endometriosis, but MRI is increasingly being used, particularly when sonographic findings are unclear, when deep pelvic endometriosis is suspected or when surgery is planned, as it provides better contrast resolution, multiplanar capabilities and a larger field of view compared to ultrasound ${ }^{(9)}$.

Jill et al. (10) reported that endometriosis, almost exclusively affects women during their reproductive years. It accounts for $20 \%$ of infertile females. In our study, the age group of women was between 22 and 48 years and 6/30 (20\%) complaining of infertility.

In our study, the ovarian involvement of 22 patients out of 25 (88\%) was markedly higher than Kruger et al. ${ }^{(11)}$ who reported ovarian involvement in 80 of 152 patients (52.6\%).

In our patient population, anterior abdominal wall endometriotic implants were detected in 2 out of 25 patients $(8 \%)$ which was higher than Kruger et al. ${ }^{(11)}$ who reported abdominal wall involvement in 4 of 152 patients $(2.6 \%)$.

In our study, implants were detected in 3 of 25 patients (12\%) which was markedly lower than Zanardi et al. ${ }^{(12)}$ who reported detection of implants in 20 out of 44 patients (45.5\%).

In our study, there were 6 out of 25 cases $(24 \%)$ showing relatively homogeneous high signal intensity on T1WI \& on T2WI, 8 out of 25 cases $(32 \%)$ showed high signal intensity on T1WI whereas on T2WI they showed intermediate signal intensity, 4 out of 
25 cases (16\%) showed high signal intensity on T1WI while on T2WI they showed low signal intensity. This agreed with the study by Imaoka et $\boldsymbol{a l} .{ }^{(13)}$ which concluded that The diagnostic MRI findings for ovarian endometriomas were (a) adnexal cysts of high signal intensity on both T1- and T2-weighted images or (b) high signal intensity on T1weighted images and intermediate or low signal intensity on T2-weighted images (shading) the dense concentration of cyclic hemorrhage and the high viscosity of the contents in the endometrioma cause T2 shortening and produce shading.

Our main differential diagnosis with endometriomas was dermoid cysts. On MRI they show high signal on $\mathrm{T} 1$, which is differentiated by signal dropout on fat suppression images, compared to endometrioma that does not suppress on $\mathrm{T} 1$ fat suppressed images. This was in agreement with Iyer and Lee ${ }^{(14)}$, who stated that endometriomas don't suppress on fat suppression, unlike a dermoid which has signal dropout on fat suppression images.

This also agreed with the study by Imaoka et al. (13) who reported that T1weighted sequences with fat suppression accentuate the spontaneous hypersignal of hemorrhagic lesions relative to the fatty tissue.

In our study The Sensitivity, Specificity, positive predictive value (PPV), negative predictive value (NPV) and accuracy of MRI in diagnosing endometriosis were $95.65 \%, 57.14 \%, 88 \%, 80 \%$, and $86.7 \%$ respectively. Except for our markedly lower Specificity, These results are more or less consistent with the study by Choudhary et al. (15) who reported that MRI yielded an overall sensitivity, specificity, and accuracy of $90 \%$, $98 \%$, and $96 \%$, respectively, for diagnosing endometriomas and in differentiating them from other gynecological masses.

\section{CONCLUSION}

Ultrasound should be the first imaging modality used in women with pelvic symptoms. It is sufficient to characterize most endometriotic cysts. If there are atypical ultrasound or clinical features, MRI will be the best problem-solving tool as in cases of indeterminate adnexal findings on sonography, when deep infiltrating endometriosis is suspected, or for presurgical mapping.

\section{REFERENCES}

1. Bulun S (2009): Endometriosis. The New England Journal of Medicine, 360(3):268-279.

2. Meuleman C, Vandenabeele B, Fieuws S et al. (2009): High prevalence of endometriosis in infertile women with normal ovulation and normospermic partners. Fertility and Sterility, 92(1):68-74.

3. Chapron C, Dubuisson J, Pansini $\mathrm{V}$ et al. (2002): Routine clinical examination is not suffcient for diagnosing and locating deeply infltrating endometriosis. The Journal of the American Association of Gynecologic Laparoscopists, 9(2):115-119.

4. Clement $P$ (2007): The pathology of endometriosis: A survey of the many faces of a common disease emphasizing diagnostic pitfalls and unusual and newly appreciated aspects. Advances in Anatomic Pathology, 14(4):241-60.

5. Bazot M, Bharwani $\mathrm{N}$, Huchon $\mathrm{C}$ et al. (2016): European society of urogenital radiology (ESUR) guidelines: MR imaging of pelvic endometriosis. European Radiology, 27(7): 2765-2775.

6. Coutinho A, Bittencourt $\mathrm{L}$, Pires $\mathrm{C}$ et al. (2011): MR imaging in deep pelvic endometriosis: A pictorial essay. Radiographics, 31:549-567.

7. Bazot M, Darai E, Hourani R et al. (2004): Deep pelvic endometriosis: MR imaging for diagnosis and prediction of extension of disease. Radiology, 232(2):379-389.

8. Woodward $P$, Sohaey $R$ and Mezzetti $T$ (2001): Endometriosis: Radiologic-Pathologic Correlation. Radiographics, 21(1):193-216.

9. Bourgioti C, Preza O, Panourgias E et al. (2017): MR imaging of endometriosis: Spectrum of disease. Diagnostic and interventional imaging, 98(11):751-767.

10. Jill A, Courtney A, Lazarus E et al. (2009): Female infertility: a systematic approach to radiologic imaging and diagnosis. Radiographics, 29 (5):1353-1370. 
11. Krüger $K$, Behrendt $K$, NiedobitekKreuter G et al. (2013): Location-dependent value of pelvic MRI in the preoperative diagnosis of endometriosis. European Journal of Obstetrics \& Gynecology and Reproductive Biology, 169(1): 93-98.

12. Zanardi $R$, Del Frate $C$, Zuiani $C$ et al. (2006): Staging of pelvic endometriosis based on MRI findings versus laparoscopic classification according to the American Fertility Society. Abdominal imaging, 28(5):733-742.

13. Imaoka I, Wada A, Matsuo M et al. (2003): MR imaging of disorders associated with female infertility: use in diagnosis, treatment, and management. Radiographics, 23(6): 1401-1421.
14. Iyer V and Lee S (2010): MRI, CT, and $\mathrm{PET} / \mathrm{CT}$ for ovarian cancer detection and adnexal lesion characterization. American Journal of Roentgenology, 194(2): 311-321.

15. Choudhary S, Fasih $\mathrm{N}$, Papadatos $\mathrm{D}$ et al. (2009): Unusual imaging appearances of endometriosis. American Journal of Roentgenology, 192(6): 1632-1644. 